
de cordeiros tratados com rBST (Somatotropina Bovina Recombinante). PUBVET, Londrina, V. 8, N. 18, Ed. 167, Art. 1780, Setembro, 2014.

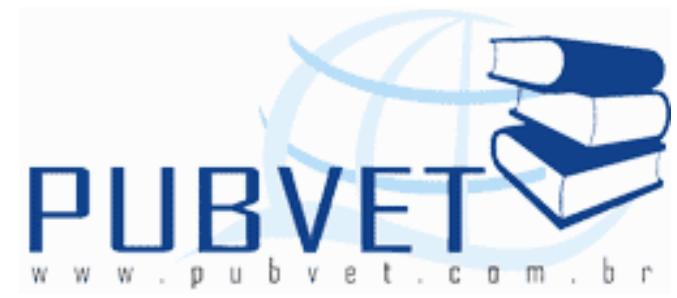

PUBVET, Publicações em Medicina Veterinária e Zootecnia.

\title{
Avaliação das características clínicas, morfométricas e do perfil bioquímico de cordeiros tratados com rBST (Somatotropina Bovina Recombinante)
}

Valéria Caobianco Sant'ana ${ }^{1}$, Robson Oliveira dos Santos², Edson Rodrigues², Tamiris Franke Dambit ${ }^{2}$, Márcio Augusto Ferreira ${ }^{1}$

${ }^{1}$ Professor(a) doutor(a) da Universidade Guarulhos

${ }^{2}$ Médico(a) veterinário(a) autônomo(a)

\section{Resumo}

A somatotropina é um hormônio conhecido por efeitos no metabolismo geral, com atuação nos tecidos moles e ósseos, na retenção de nitrogênio, e na produção de leite. Neste estudo foram utilizados 47 cordeiros da raça Santa Inês, sendo que 27 foram tratados com 2 aplicações de $250 \mathrm{mg}$ de BOOSTIN ${ }^{\circledR}$ em torno dos 35 e 55 dias de idade e foram comparados a 20 cordeiros que receberam placebo. Avaliações clínicas, morfométricas, bioquímicas e o peso foram realizadas antes e quinzenalmente após o tratamento. De acordo com os resultados concluiu-se que o protocolo não influenciou o peso dos animais nem mesmo as medidas morfométricas e perfil bioquímico. O uso da somatotropina não causou qualquer efeito adverso na saúde dos cordeiros bem como alterações no local da aplicação. Palavras-chave: Somatotropina; Bioquímico; Ovinos; rBST; Crescimento. 

de cordeiros tratados com rBST (Somatotropina Bovina Recombinante). PUBVET, Londrina, V. 8, N. 18, Ed. 167, Art. 1780, Setembro, 2014.

\title{
Evaluation of clinical, morphometric and biochemical profile of treated lambs with rBST (Recombinant Bovine Somatotropin)
}

\begin{abstract}
Somatotropin is a proteinic hormone known for countless effects in the general metabolism of the body, as the increase in the soft and osseous tissues and the stimulus to the production of milk. For the present study 47 lambs of the breed "Santa Inês" were used. They were from the same place and were divided into two groups: one treatment-group $(n=27)$, which was treated with two applications of $250 \mathrm{mg}$ of rBST (Boostin $\AA$ ), via subcutaneous in the ischium-retal region. The average age were 35 to 41 days old (M1) and 55 and 61 days old (M2). The control-group ( $n=20$ ) received injections of physiological solution in suitable content. Clinical, morphometric, weight and biochemical evaluations were performed before the treatment and fortnightly. According to the results observed it was possible to conclude that the treatment has not influenced the gaining of weight of the animals, not even the measure used in morphometry. The usage of rBST has not caused any side effect in the health of the lambs or any alteration in the place of the application. The biochemical profile of the animals has not suffered any changes that could be connected to the treatment.
\end{abstract} Keywords: Somatotropin; Biochemical; Ovine; rBST; Development.

\section{INTRODUÇÃO}

O hormônio de crescimento (GH) ou somatotropina (ST) é um hormônio protéico, sintetizado e secretado pela glândula pituitária anterior (RIGHI, 2006; RANGEL et al. 2008) e assim como qualquer alimento protéico consumido por via oral, é digerido por enzimas (BAUMAN, 1992). Trata-se de um dos mais importantes reguladores do metabolismo geral do corpo, do processo de síntese 
SANT'ANA, V.C. et al. Avaliação das características clínicas, morfométricas e do perfil bioquímico de cordeiros tratados com rBST (Somatotropina Bovina Recombinante). PUBVET, Londrina, V. 8, N. 18, Ed. 167, Art. 1780, Setembro, 2014.

do leite, do crescimento, da composição do ganho de peso e influi na eficiência reprodutiva (RANGEL et al. 2008).

O efeito anabólico da somatotropina é caracterizado pelo aumento nos tecidos moles e ósseos, aumento da retenção de nitrogênio, além de estimular a produção de leite, principalmente em bovinos (NOGUEIRA, 2006).

A somatotropina bovina recombinante (rBST) é um hormônio peptídeo sintetizado através de DNA recombinante (RIGHI, 2006). Quando administrada aos ruminantes, causa uma série de respostas em diferentes tecidos do organismo. Dentre eles, estimula a síntese de proteína, glicose e atua na eficiência parcial da utilização dos aminoácidos, nos ossos pelo acréscimo mineral paralelamente ao crescimento tecidual, e na oxidação de gordura, inibe o transporte de glicose para o tecido periférico e desencadeia, em animais lactantes, o efeito homeorrético (RANGEL et al, 2008; SANT'ANA, 2010).

Amorim et al (2007) observaram aumento nas concentrações séricas de colesterol, proteína total e plasmáticas de glicose em touros Nelore tratados com rBST, enquanto Castro et al (2002), concluíram que a rBST não interfere no metabolismo intermediário do colesterol e da glicose.

O objetivo desse trabalho foi avaliar a possível influência de duas aplicações de 250 mg de rBST em cordeiros de 35 a 40 dias, em intervalos de 15 dias entre as aplicações, sobre o ganho de peso e constituintes da bioquímica sanguínea.

\section{MATERIAL E MÉTODOS}

Para compor os grupos experimentais foram utilizados 47 cordeiros (machos e fêmeas) da raça Santa Inês de um mesmo padrão genético nascidos em uma propriedade em Atibaia - SP, submetidos ao mesmo sistema de criação. Ao início do experimento os ovinos tinham de 15 a 21 dias de idade e foram divididos de forma aleatória em dois grupos experimentais (grupo tratado e controle). 
SANT'ANA, V.C. et al. Avaliação das características clínicas, morfométricas e do perfil bioquímico de cordeiros tratados com rBST (Somatotropina Bovina Recombinante). PUBVET, Londrina, V. 8, N. 18, Ed. 167, Art. 1780, Setembro, 2014.

No grupo tratado $(n=27)$ os cordeiros receberam duas aplicações de $250 \mathrm{mg}$ de somatotropina (BOOSTIN ${ }^{\circledR}$ ) por via sub-cutânea, na região ísqueo-retal. Os tratamentos foram realizados quando os animais tinham entre 35 e 41 dias (M1) e entre 55 e 61 dias de idade (M2), sempre se alternando os lados da aplicação.

Para os animais do grupo-controle $(n=20)$, foram efetuados os mesmos procedimentos e, para efeito de comparação, foram administradas injeções de solução fisiológica, em volume correspondente.

Parâmetros clínicos (frequência cardíaca, frequência respiratória, temperatura corpórea, apetite) foram avaliados nos dias dos tratamentos e 24 horas após os animais foram monitorados em relação à atitude e apetite. Foram observadas, também, possíveis alterações no local da administração do medicamento (dor, inchaço, alopecia e outras).

As características morfométricas nos animais constituintes dos dois grupos experimentais, foram realizadas nos seguintes momentos: $M 0=15$ a 21 dias de idade; $M 1=35$ a 41 dias; $M 2=55$ a 61 dias; $M 3=75$ a 81 dias. Enquanto a coleta de amostras do soro sanguíneo para pesquisa do perfil bioquímico foram realizadas da seguinte forma: $M 1=35$ a 41 dias; $M 2=55$ a 61 dias; $M 3=75$ a 81 dias.

Para avaliação morfométrica, as seguintes medidas foram consideradas: altura na cernelha, altura na garupa, comprimento de corpo, perímetro de tórax, perímetro de abdômen, comprimento de garupa, largura de garupa, perímetro escrotal e peso. Uma fita métrica foi utilizada para determinação das medidas, exceto peso que foi obtido por uma balança.

As amostras de soro sanguíneo obtidas por venopunção jugular foram destinadas ao Laboratório de Análises Clínicas do Hospital Veterinário da Universidade Guarulhos para realização das provas bioquímicas, utilizando-se as técnicas de rotina preconizadas pelo laboratório. Foram avaliados AST (aspartato 
SANT'ANA, V.C. et al. Avaliação das características clínicas, morfométricas e do perfil bioquímico de cordeiros tratados com rBST (Somatotropina Bovina Recombinante). PUBVET, Londrina, V. 8, N. 18, Ed. 167, Art. 1780, Setembro, 2014.

amino tranferase), GGT (gama glutamil transferase), uréia, creatinina, glicose, bilirrubinas, albumina, proteína total e triglicérides.

\subsection{Análise estatística}

Para análise estatística foram utilizados testes paramétricos (t de Student) para as variáveis como dosagens bioquímicas, peso, altura e largura da garupa.

\section{RESULTADOS}

De acordo com a análise dos resultados obtidos, foi possível concluir que o protocolo utilizado de duas aplicações de $250 \mathrm{mg}$ de rBST em cordeiros em torno dos 40 e 60 dias de idade não influenciou o ganho de peso dos animais. Também não foram evidentes alterações clínicas gerais e no local da aplicação. O perfil bioquímico avaliado não sofreu influência do tratamento em comparação aos animais do grupo controle.

Os resultados gerais foram demonstrados nas Tabelas 1 e 2 para melhor poderem ser apreciados. 


\section{Tabela 1 - Características morfométricas $(\mathrm{em} \mathrm{cm})$ e peso $(\mathrm{em} \mathrm{kg})$ de cordeiros Santa Inês tratados com somatotropina (GT) e grupo controle (GC) representados por médias e desvios padrão. São Paulo, 2010.}

\begin{tabular}{|c|c|c|c|c|c|c|c|c|}
\hline \multirow{2}{*}{$\begin{array}{c}\text { Parâmetro } \\
\text { s }\end{array}$} & \multicolumn{2}{|c|}{ MO } & \multicolumn{2}{|c|}{ M1 } & \multicolumn{2}{|c|}{ M2 } & \multicolumn{2}{|c|}{ M3 } \\
\hline & GT & GC & GT & GC & GT & GC & GT & GC \\
\hline $\begin{array}{c}\text { Peso (em } \\
\mathrm{Kg})\end{array}$ & $\begin{array}{c}8,42 \pm \\
2,44\end{array}$ & $\begin{array}{c}8,72 \pm \\
3,33\end{array}$ & $\begin{array}{c}11,80 \pm \\
3,06\end{array}$ & $\begin{array}{c}11,70 \pm \\
4,18\end{array}$ & $\begin{array}{c}14,98 \pm \\
3,60\end{array}$ & $\begin{array}{c}15,24 \pm \\
4,97\end{array}$ & $\begin{array}{c}18,30 \pm \\
4,12\end{array}$ & $18,98 \pm 4,93$ \\
\hline $\begin{array}{l}\text { Perímetro } \\
\text { torácico }\end{array}$ & $\begin{array}{c}48,19 \pm \\
5,52\end{array}$ & $\begin{array}{c}48,33 \pm \\
6,81\end{array}$ & $\begin{array}{c}53,85 \pm \\
4,78\end{array}$ & $\begin{array}{c}52,86 \pm \\
6,75\end{array}$ & $\begin{array}{c}59,83 \pm \\
3,93\end{array}$ & $\begin{array}{c}58,85 \pm \\
5,68\end{array}$ & $\begin{array}{c}64,21 \pm \\
3,54\end{array}$ & $63,58 \pm 4,71$ \\
\hline $\begin{array}{l}\text { Perímetro } \\
\text { abdominal }\end{array}$ & $\begin{array}{c}52,74 \pm \\
8,07\end{array}$ & $\begin{array}{c}52,24 \pm \\
9,68\end{array}$ & $\begin{array}{c}60,07 \pm \\
6,99\end{array}$ & $\begin{array}{c}59,00 \pm \\
8,99\end{array}$ & $\begin{array}{c}66,67 \pm \\
5,42 \\
\end{array}$ & $\begin{array}{c}66,45 \pm \\
6,73\end{array}$ & $\begin{array}{c}72,83 \pm \\
4,69\end{array}$ & $72,53 \pm 5,51$ \\
\hline $\begin{array}{c}\text { Comprime } \\
\text { nto do } \\
\text { corpo }\end{array}$ & $\begin{array}{c}41,56 \pm \\
5,65\end{array}$ & $\begin{array}{c}42,43 \pm \\
6,76\end{array}$ & $\begin{array}{c}46,85 \pm \\
4,67\end{array}$ & $\begin{array}{c}46,24 \pm \\
5,49\end{array}$ & $\begin{array}{c}50,92 \pm \\
4,74\end{array}$ & $\begin{array}{c}50,70 \pm \\
5,83\end{array}$ & $\begin{array}{c}54,54 \pm \\
3,80\end{array}$ & $54,68 \pm 4,71$ \\
\hline $\begin{array}{l}\text { Altura da } \\
\text { cernelha }\end{array}$ & $\begin{array}{c}47,67 \pm \\
5,19\end{array}$ & $\begin{array}{c}49,24 \pm \\
5,43\end{array}$ & $\begin{array}{c}51,77 \pm \\
4,65\end{array}$ & $\begin{array}{c}52,33 \pm \\
5,22\end{array}$ & $\begin{array}{c}56,46 \pm \\
4,24\end{array}$ & $\begin{array}{c}57,20 \pm \\
4,91\end{array}$ & $\begin{array}{c}59,54 \pm \\
5,04\end{array}$ & $59,95 \pm 4,60$ \\
\hline $\begin{array}{l}\text { Altura da } \\
\text { garupa }\end{array}$ & $\begin{array}{c}49,26 \pm \\
4,74\end{array}$ & $\begin{array}{c}50,71 \pm \\
5,71\end{array}$ & $\begin{array}{c}53,04 \pm \\
4,45\end{array}$ & $\begin{array}{c}53,86 \pm \\
5,63\end{array}$ & $\begin{array}{c}57,17 \pm \\
4,08\end{array}$ & $\begin{array}{c}58,40 \pm \\
4,86\end{array}$ & $\begin{array}{c}60,96 \pm \\
4,71\end{array}$ & $61,26 \pm 4,68$ \\
\hline $\begin{array}{c}\text { Comprime } \\
\text { nto da } \\
\text { garupa }\end{array}$ & $\begin{array}{c}12,44 \pm \\
2,29 \\
\end{array}$ & $\begin{array}{c}13,33 \pm \\
2,58 \\
\end{array}$ & $\begin{array}{c}13,52 \pm \\
1,63\end{array}$ & $\begin{array}{c}13,95 \pm \\
2,16\end{array}$ & $\begin{array}{c}14,83 \pm \\
1,90\end{array}$ & $\begin{array}{c}14,55 \pm \\
1,93\end{array}$ & $\begin{array}{c}15,29 \pm \\
1,49\end{array}$ & $15,21 \pm 1,55$ \\
\hline $\begin{array}{c}\text { Largura } \\
\text { da garupa }\end{array}$ & $\begin{array}{c}14,07 \pm \\
2,54\end{array}$ & $\begin{array}{c}14,62 \pm \\
2,38\end{array}$ & $\begin{array}{c}15,93 \pm \\
2,23\end{array}$ & $\begin{array}{c}15,81 \pm \\
2,93\end{array}$ & $\begin{array}{c}19,08 \pm \\
2,17\end{array}$ & $\begin{array}{c}18,15 \pm \\
2,52\end{array}$ & $\begin{array}{c}21,17 \pm \\
2,65\end{array}$ & $20,37 \pm 2,31$ \\
\hline $\begin{array}{c}\text { Perímetro } \\
\text { escrotal }\end{array}$ & $\begin{array}{c}8,36 \pm \\
1,45\end{array}$ & $\begin{array}{c}9,25 \pm \\
1,71\end{array}$ & $\begin{array}{c}10,43 \pm \\
1,28\end{array}$ & $9,46 \pm 2,70$ & $\begin{array}{c}11,92 \pm \\
1,56\end{array}$ & $\begin{array}{c}11,31 \pm \\
2,66\end{array}$ & $\begin{array}{c}13,33 \pm \\
2,23\end{array}$ & $13,42 \pm 3,40$ \\
\hline
\end{tabular}


SANT'ANA, V.C. et al. Avaliação das características clínicas, morfométricas e do perfil bioquímico de cordeiros tratados com rBST (Somatotropina Bovina Recombinante). PUBVET, Londrina, V. 8, N. 18, Ed. 167, Art. 1780, Setembro, 2014.

Tabela 2 - Perfil bioquímico de cordeiros Santa Inês tratados com somatotropina (GT) e grupo controle (GC) representados por médias e desvios padrão. São Paulo, 2010.

\begin{tabular}{|c|c|c|c|c|c|c|}
\hline Perfil & M1 & & M2 & & M3 & \\
\hline bioquímico & GT & GC & GT & GC & GT & GC \\
\hline AST $(U / I)$ & $82,00 \pm 21,54$ & $87,81 \pm 42,85$ & $86,83 \pm 16,97$ & $94,25 \pm 29,69$ & $81,78 \pm 22,42$ & $91,16 \pm 20,11$ \\
\hline GGT (U/I) & $66,04 \pm 18,70$ & $65,24 \pm 12,19$ & $71,38 \pm 29,71$ & $64,00 \pm 15,00$ & $68,42 \pm 22,97$ & $61,89 \pm 8,78$ \\
\hline Creatinina (mg/dl) & $0,57 \pm 0,09$ & $0,54 \pm 0,09$ & $0,58 \pm 0,06$ & $0,57 \pm 0,07$ & $0,59 \pm 0,03$ & $0,59 \pm 0,05$ \\
\hline Uréia (mg/dl) & $19,81 \pm 4,42$ & $21,43 \pm 5,49$ & $19,00 \pm 4,83$ & $20,65 \pm 4,20$ & $20,54 \pm 3,66$ & $21,89 \pm 4,05$ \\
\hline Albumina (g/dl) & $2,84 \pm 0,32$ & $2,96 \pm 0,51$ & $2,90 \pm 0,42$ & $2,99 \pm 0,28$ & $2,90 \pm 0,33$ & $3,11 \pm 0,49$ \\
\hline Proteínas totais ( $\mathrm{g} / \mathrm{dl}$ ) & $5,42 \pm 0,84$ & $7,83 \pm 0,96$ & $5,49 \pm 0,51$ & $5,62 \pm 0,50$ & $5,67 \pm 0,63$ & $5,62 \pm 0,35$ \\
\hline Colesterol (mg/dl) & $62,50 \pm 21,03$ & $69,14 \pm 19,98$ & $61,13 \pm 12,77$ & $68,85 \pm 15,93$ & $66,79 \pm 14,44$ & $69,11 \pm 16,83$ \\
\hline Triglicérides (mg/dl) & $76,48 \pm 16,94$ & $80,33 \pm 35,43$ & $80,03 \pm 41,39$ & $72,65 \pm 9,08$ & $81,92 \pm 12,35$ & $70,26 \pm 10,97$ \\
\hline
\end{tabular}


SANT'ANA, V.C. et al. Avaliação das características clínicas, morfométricas e do perfil bioquímico de cordeiros tratados com rBST (Somatotropina Bovina Recombinante). PUBVET, Londrina, V. 8, N. 18, Ed. 167, Art. 1780, Setembro, 2014.

\section{DISCUSSÃO}

Os estudos sobre os efeitos da rBST no metabolismo de ruminantes vem sendo realizados por diversos pesquisadores do mundo e do Brasil (BAUMAN, 1992; RIGHI; 2006) com diferentes protocolos e resultados. Embora alguns estudiosos terem afirmado a influência positiva do hormônio sobre o crescimento em desenvolvimento em ruminantes (RANGEL et al. 2008), ovinos tratados neste trabalho com duas doses de $250 \mathrm{mg}$ aos 35 e 55 dias de idade nenhuma influência do tratamento sobre o crescimento foi observada. MATOS (1998) relata em sua pesquisa que nenhum aumento da incidência de mastite e debilidade dos animais foi observado diferindo. Resultados semelhantes em bovinos foram observados por (Nascimento et al. 2003) ao avaliarem ganho de peso, conversão alimentar e rendimento de carcaça de novilhas mestiças de Nelore e Red Angus tratadas tanto com dose única de $250 \mathrm{mg}$ de rBST quanto tratadas quinzenalmente.

Os resultados obtidos na presente pesquisa estão de acordo aos encontrados por (Moreira et al. 2000) que não observaram influência do tratamento com rBST quanto ao ganho de peso dos animais tratados.

A avaliação dos resultados deste estudo permitiu a afirmação de que o tratamento de duas doses de $250 \mathrm{mg}$ em cordeiros não afetou os parâmetros bioquímicos nem mesmo a saúde dos animais, estando de acordo com os resultados descritos por (Castro et a.l 2002) que também não identificaram influência do uso de rBST nos níveis séricos do colesterol e de glicose em ovelhas. Por outro lado, nas condições experimentais estudadas por (Amorim et al. 2007), a administração de rBST em touros jovens e adultos da raça Nelore altera os níveis de colesterol total sérico, glicose plasmática, ácidos graxos nãoesterificados, proteínas totais sérica e uréia sérica em bovinos de corte indianos. 
SANT'ANA, V.C. et al. Avaliação das características clínicas, morfométricas e do perfil bioquímico de cordeiros tratados com rBST (Somatotropina Bovina Recombinante). PUBVET, Londrina, V. 8, N. 18, Ed. 167, Art. 1780, Setembro, 2014.

\section{CONCLUSÃO}

Atualmente existem diversas pesquisas realizadas com rBST em todo o mundo, porém em sua maioria voltada para produção de leite. Isso talvez se dê pelo fato de fornecedores de rBST incentivarem pesquisas voltadas para essa área já que a indicação dos produtos disponíveis atualmente seja para o aumento da lactação de vacas leiteras.

As avaliações realizadas em pequenos ruminantes são escassas e resultados encontrados parecem ser inconclusivos.

Os resultados observados no presente trabalho demonstraram que a administração de duas aplicações de $250 \mathrm{mg}$ de rBST aos cordeiros de 35 e 55 dias de idade não modificou as características morfométricas e peso quando comparados aos animais não tratados. As observações clínicas e dosagens bioquímicas permitem afirmar que o tratamento de cordeiros com rBST não acarretou em dano à saúde dos animais.

Com base na atual pesquisa realizada, estudos futuros talvez devam lançar mão de dosagens diferentes das empregadas nesse e na maioria dos trabalhos.

\section{REFERÊNCIAS}

AMORIM, L.S.; TORRES, C.A.A.; MORAES, E.A.; SILVA FILHO, J.M.; GUimARÃES, J.D. Perfil metabólico de touros da raça Nelore (Bos taurus indicus) confinados e tratados com somatotrofina bovina recombinante (r-bST) Arq. Bras. Med. Vet. Zootec., v.59, n.2, p.434-442, 2007;

BAUMAN, D.E. 1992 Bovine somatotropin: Review of an emerging animal technology. J. Dairy Sci., 75:3432-3451, 1992;

CASTRO, A.A.P.; NORO, M.; CECIM, M.N.M. A somatotrofina bovina recombinante sobre a ovulação, condição corporal e níveis séricos de colesterol e glicose em ovinos. Ciência Rural, Santa Maria, v.32, n.3, p.479-483, 2002.

MATTOS, w. Somatotropina na pecuária de leite e de corte. In: ANUAL DA SOCIEDADE BRASILEIRA DE ZOOTECNIA, p. 35-52. 
NASCIMENTO, W.G.; PRADO, I.N.; RIGOLON, L.P.; MARQUES, J.A.; WADA, F.Y.; MATSISHITA, M.; SCOMPARIN, V.X. Somatotropina Recombinante (rBST) sobre o Desempenho e a Digestibilidade Aparente de Novilhas ( $1 / 2$ Nelore x $1 / 2$ Red Angus) em Confinamento R. Bras. Zootec., v.32, p. 456-464, 2003.

NOGUEIRA, G.P. Farmacologia do eixo hipotálamo-hipófise In: SPINOSA, H.S.; GÓRNIAK, S.L.; BERNARDI, M.M. Farmacologia Aplicada à Medicina Veterinária - $4^{a}$ Ed. - Rio de Janeiro: Guanabara Koogan, 2006, Cap. 29, p. 375-376.

RANGEL, A.H.N.; DIVINO, M.R.; LEONEL, F.P. JUNIOR, D.M.L. Somatotropina na pecuária de leite PUBVET, V.2, N.22, Art \#245, Jun 1, 2008.

RIGHI, D.A.; PALERMO-NETO, J. Somatotropina Bovina In: SPINOSA, H.S.; GÓRNIAK, S.L.; BERNARDI, M.M. Farmacologia Aplicada à Medicina Veterinária - $4^{a}$ Ed. - Rio de Janeiro: Guanabara Koogan, 2006, Cap. 51, p. 628,629,631.

SANT'ANA, V.A.C. Perspectivas do uso da somatotropina (BST) em pequenos ruminantes FEINCO, 2010. 